\title{
Management of Complications in Endoscopic Endonasal Surgery for Pituitary Adenomas
}

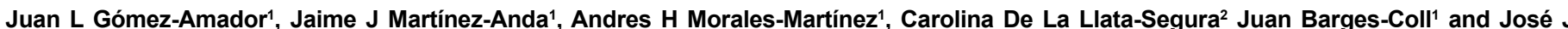
Martínez-Manrique ${ }^{1 *}$

${ }^{1}$ Department of Neurological Surgery, Instituto Nacional de Neurología y Neurocirugía, Mexico City, Mexico

${ }^{2}$ Department of otolaryngology, Hospital Español de México, Mexico City, Mexico

\begin{abstract}
Objective: To describe a case series of patients with pituitary adenomas with endoscopic endonasal transsphenoidal approach, the technique performed and complications in our centre.

Methods: The technique performed in a series of 98 consecutive patients, and description of their complications and the protocol followed to treat these complications.

Results: 120 patients where submitted to Skull Base Endoscopic Surgery, of which $98(81.66 \%)$ had diagnosis of non-functioning pituitary adenoma in a two year period. Complications described are postoperative cerebrospinal fluid leaks $(6.12 \%)$, vascular injury $(3.06 \%)$ and cranial nerve injury $(2.04 \%)$.

Conclusion: A specific protocol is followed in our institution to treat different complications in pituitary adenoma transnasal endoscopic surgery in order to improve outcomes. Prompt and diligent identification of complications is of utmost importance for their treatment.
\end{abstract}

Keywords: Endonasal approach; Endoscopic surgery; Skull base surgery; Complication; Safety; Pituitary adenoma

\section{Introduction}

Minimally invasive procedures in neurosurgery, during the last 2 decades have become a constant subject of update and evolution [1]. Skull Base Surgery as other disciplines has adopted this approach with the advent of Endoscopic Endonasal Anterior approach [2]. The origin of this approach to the sella turcica has been attributed to the work of researchers like Carrau, Jho, Cappabianca, Frank among others [2-4].

\section{Methods}

We describe a case series of patients operated in the Neurosurgery Department of our institution with diagnosis of Non-functioning Pituitary Adenoma, through Transnasal Endoscopic Surgery in the period between 2013 and 2014 in conjunction with the Otolaryngology department. All the patients were protocolled with preoperative Magnetic Resonance (Figure 1), Computed Tomography for Neuronavigation using BranLab $^{\star}$ for skull and paranasal sinuses, endocrinology lab work-up and in cases necessary, suppressive hormone tests for Cushing's and Acromegaly. In patients with lesions invading the Cavernous Sinus (Figure 2) with a Knosp classification $>2$ intraoperative monitoring for $3 \mathrm{rd}$ and 6th cranial nerves on the affected side was performed.

Analysis for epidemiologic variables, preoperative hormone status, tumour grading using Hardy-Vezina [5] (HV) and Knosp [6] classifications, intraoperative findings, surgical complications, postoperative follow-up with laboratory and imaging studies where performed.

Surgical complications were classified in the following manner: 1) Vascular Complications, with intraoperative haemorrhage by vascular injury; 2) Postoperative CSF leak, when the patient presented with liquid spill in the postoperative follow-up by the otolaryngology team, when endoscopic endonasal exam was performed; 3) Cranial Nerve injury, when the patient presented with visual impairment compared to their preoperative status, or presented with ophthalmoplegia; 4) Panhypopituitarism, when the patient required hormonal replacement in more than 2 hormonal axis, when there was no preoperative hormonal deficit.

\section{Surgical technique}

Patients in the operating room where placed in dorsal decubitus, fixated with a Mayfield ${ }^{\oplus}$ headholder, neuronavigation protocol was performed, and the head was rotated to the right with a 10 degree cervical extension. With the help of an endoscope (Storz $\left.{ }^{\circledR}\right)$ the medial turbinates and nasal septum were infiltrated with $2 \%$ lidocaineepinephrine solution; the nasal septum was luxated (Figure 3) to broaden the corridor, after which dissection of the nasal septal mucosa with vascular pedicle of the posterior septal artery for the nasoseptal flap was performed. The nasoseptal flap is then placed upon the nasopharynx. This last step was accomplished in selected cases in which an anticipated risk for intraoperative fistula was expected (HV $>$ IIIC or Knosp > 3). Then a posterior septostomy, resection of the sphenoidal rostrum and broadening of the sphenoidal sinus window was achieved until the reference points were located (sella turcica, carotid prominence, optic nerve prominence, medial and lateral carotid-optic recesses on both sides). Then the sellar floor was drilled with the following limits: sellar tubercule anteriorly, both internal carotid arteries (ICA) laterally, and the sellar dorsum posteriorly.

*Corresponding author: José de Jesús Martínez Manrique, Department of Neurological Surgery, Instituto Nacional de Neurología y Neurocirugía, Mexico City, Mexico, Tel: +52 1559199 4845; E-mail: jesus.martinez@neurocirugia-innn. com

Received January 07, 2015; Accepted January 28, 2015; Published January 30, 2015

Citation: Gómez-Amador JL, Martínez-Anda JJ, Morales-Martínez AH, LlataSegura CDL, Barges-Coll J, et al. (2015) Management of Complications in Endoscopic Endonasal Surgery for Pituitary Adenomas. J Neurol Disord 3: 209doi: 10.4172/2329-6895.1000209

Copyright: () 2015 Gómez-Amador JL, et al. This is an open-access article distributed under the terms of the Creative Commons Attribution License, which permits unrestricted use, distribution, and reproduction in any medium, provided the original author and source are credited. 


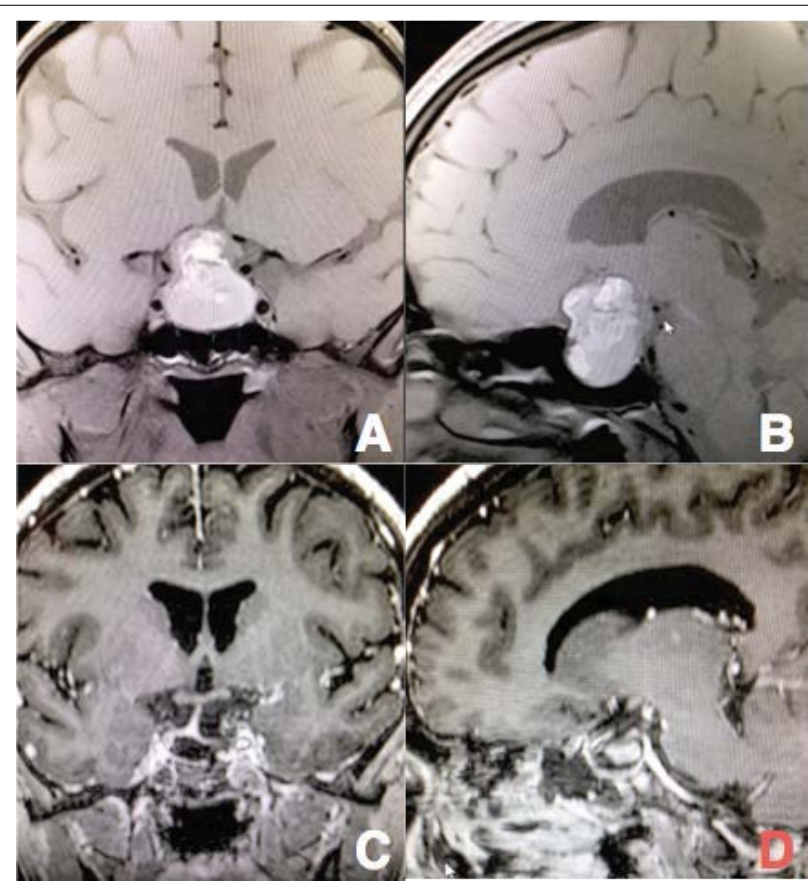

Figure 1: $A$ and $B$ : Coronal $(A)$ and sagittal $(B)$ contrast-enhanced $M R$ images showing preoperative images of a macroadenoma with suprasellar extension. C and D: Postoperative coronal (C) and sagittal (D) MR images demonstrating complete resection of the lesion and preservation of the pituitary gland at the right side of the sella.

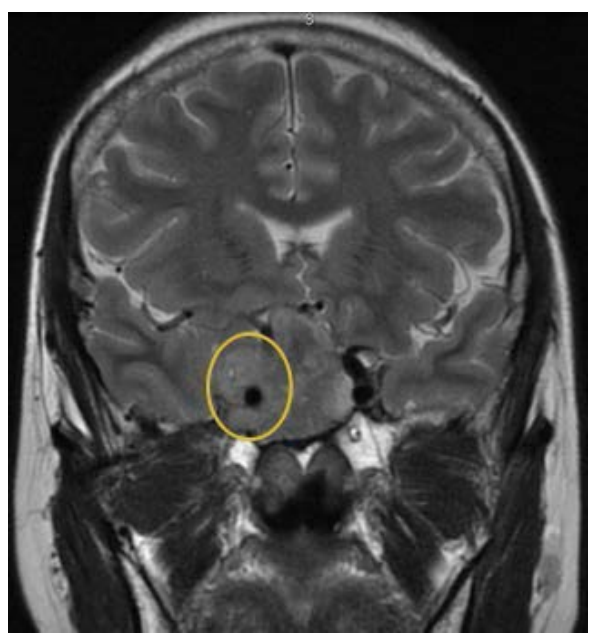

Figure 2: Magnetic resonance of a patient with a pituitary adenoma with cavernous sinus invasion.

In patients with cavernous sinus invasion (Figure 2) the approach was extended laterally and anteriorly to the sphenoidal planum. Then durotomy was performed in an " $\mathrm{x}$ " or “+” fashion after which extracapsular dissection of the lesion what achieved. Once dissected the lesion was debulked centrally with Rail's spoon, Hardy dissectors and aspiration. Then thorough inspection of remaining glandular tissue and arachnoid from the suprasellar cistern was accomplished. Then the reconstruction of the approach was performed with intradural and epidural Duragen ${ }^{\circledast}$ patch, then placement of the nasoseptal flap (accordingly) and fixation of the flap with a Foley catheter (which was then removed on postoperative day 5).

\section{Results}

\section{Patients}

A total of 167 patients with pituitary adenoma were treated in the Neurosurgery Department at the Instituto Nacional de Neurología y Neurocirugía in Mexico City during the 2-year period between 2013 and 2014. One hundred and twenty patients were submitted to transnasal endoscopic skull base surgery with non-functioning pituitary adenomas accounting for $81.66 \%$ (98) of these patients. Of these patients $58.56 \%$ were males and $41.84 \%$ were females. The average age was 45 years old $(S D \pm 2)$; average follow-up was 8 months.

\section{Clinical presentation}

The most common chief complaint before diagnosis of pituitary adenoma was visual impairment (visual field and acuity) in $48.97 \%$ (48); followed by headache in $38.77 \%$ (38), amenorrhea in $6.12 \%$ (6), ophthalmoplegia in $3.06 \%$ (3) and hydrocephalus requiring shunt in $3.06 \%$ (3). The average time between the beginning of symptoms and diagnosis was 24 months.

In the workup visual acuity was found preserved (>20/60) in $48.97 \%$ (48), impaired (20/60 - 20/400) in $20.40 \%$ (20) and amaurotic $(<20 / 400)$ in $30.61 \%$ (30) on either side. Visual fields were found with bilateral temporal hemianopia in $70.40 \%$ (69), unilateral amaurosis in $11.22 \%$ (11), no patients were found with bilateral amaurosis and with no impairment in $18.36 \%$ (18). Hormonal status was reported with thyroid dysfunction in $15.3 \%$ (15), cortisol impairment in $6.12 \%$ (6), gonadotropic impairment in $18.36 \%$ (18), and panhypopituitarism in $10.20 \%(10)$.

Anatomic analysis of the lesions: 55.1\% (54) were invasive (HV $>$ III or Knosp >2) of which $44.49 \%$ (24) invaded the cavernous sinus (Knosp > 2), 46.29\% (25) invaded the sphenoidal sinus (HV > III) and $9.25 \%$ (5) had invasion to both cavernous and sphenoidal sinuses. Tables 1 and 2 describe the distribution of patients according to HV and Knosp classifications respectively. The volumetric analysis showed and average volume of $14 \mathrm{cc}(\mathrm{SD} \pm 13)$; the dimensions of the lesions were antero-posterior $24 \mathrm{~mm}$ ( $\mathrm{SD} \pm 10.2$ ), supero-posterior $31 \mathrm{~mm}$ (SD $\pm 11.4)$ and latero-lateral $25 \mathrm{~mm}(\mathrm{SD} \pm 9)$.

\section{Intraoperative findings}

We found intraoperative CSF leak in $34.69 \%$ (34) of patients, suprasellar arachnoid membrane prolapse in $46.93 \%$ (46), a nasoseptal flap was performed in $72.44 \%$ (71) of cases and we had an incidence of $3.06 \%$ (3) for intraoperative haemorrhage. Grades of resection are described in Table 3. Figure 4 describes different situations in which CSF leak is expected.

\section{Complications}

The overall incidence of complications was $11.22 \%$ (11). The most common was postoperative CSF leak in $6.12 \%$ (6) followed by vascular injury in $3.06 \%$ (3) and cranial nerve injury in $2.04 \%$ (2). Of the vascular injuries one was to the ICA, one to the posterior communicating artery, and the other to a frontal polar branch of the anterior cerebral artery.

\section{Clinical results}

We found visual improvement in $73.46 \%$ (72) and we had one case $(1.02 \%)$ of permanent visual impairment. Ten patients $(10.20 \%)$ required surgical re-intervention for remnant tumour growth. Twenty- 


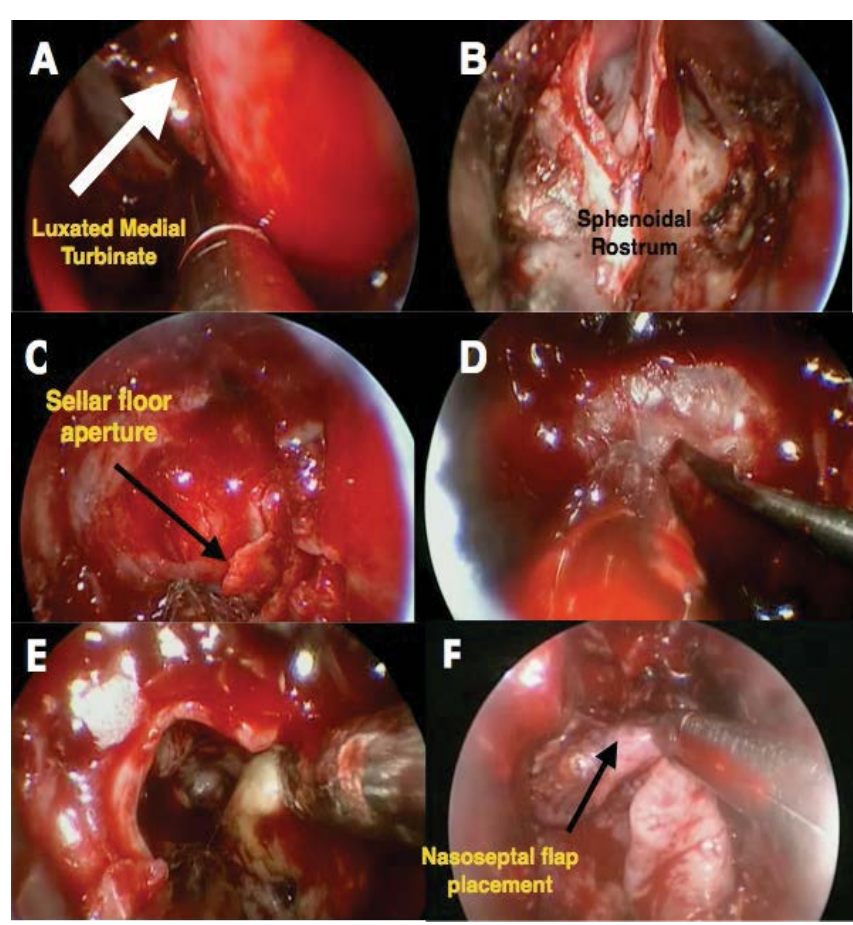

Figure 3: Surgical Technique A) Luxation or resection of medial turbinate is performed (usually right side). B) Both nasal cavities are communicated using a posterior septostomy. Sphenoidal rostrum is exposed. C) Once drilled the wall of the sphenoidal sinus the sellar floor is exposed D) Durotomy is performed in an "X" or "+" fashion. E) Central lesion debunking with Rail and Hardy dissectors. F) Sellar floor reconstruction with nasoseptal mucosa flap.

nine patients $(29.59 \%)$ were treated with fraccionated stereotactic radiotherapy ( $50.4 \mathrm{~Gy}$ in 28 sessions). We report a mortality rate of $2.04 \%$ (2) one of which was secondary to ICA injury, and the other due to sever vasospasm after intraoperative haemorrhage.

\section{Discussion}

Several authors [7-10] have described in numerous series their expertise in techniques, perioperative care and avoidance of complications in the surgical treatment of pituitary adenomas. Kassam et al. [7], described a case series of 800 patients with skull base surgery in a 9 year period. They report an incidence of $15.9 \%$ of CSF leaks, neurologic injury in $1.8 \%$ and vascular injury in $0.9 \%$. Cavallo et al. [8], described a technique used to repair the sellar floor using dural substitute and fibrin glue for reconstruction. Gondim et al. [9], recently reported a series of 50 cases in which the use of naso-septal flap for the reconstruction of the sellar floor has decreased the incidence of CSF leak. Thomas et al. [10], recently demonstrated in a small cohort study that short hospital stay for this patients is feasible with no change in postoperative outcome. Continuous work to describe complications and expertise by different surgeons globally, as well of development and deployment of protocols for the management of patients with pituitary adenomas sheds light into decreasing the incidence and establishment of early measures to treat complications. Therefore we describe here the protocol we use in our institution for the avoidance and treatment of these complications.

\section{CSF leak}

Several techniques have been described for reconstruction of the sellar floor in endoscopic skull base surgery [11-13]. In our case series we use a technique developed by Zanation et al. [14], in which they use a nasoseptal flap reconstruction for the approach. This has proved to be an excellent form of reconstruction reporting a $94 \%$ successful rate.

When we have a patient in which we suspect a CSF leak, they are submitted to the rhinologist, for endoscopic inspection. Once the diagnosis is confirmed the patient is admitted immediately to the $\mathrm{OR}$, the procedure (Figure 5) consists on a) endoscopic exploration of the previous approach; b) a Duragen ${ }^{\circ}$ patch is placed intra- and extradurally, 3) Fascia latta is harvested and fixated with a nasal septum bone flap using Beriplast ${ }^{\bullet}$ and 4) the nasoseptal pediculated flap is placed and fixated with a Foley catheter.

\section{Vascular injuries}

Although oftentimes exposure allows the surgeon to identify vascular and nervous bone prominences during the approach, in some circumstances this references are absent and vascular lesions to the cavernous sinus might be encountered [15]. The rate of vascular injuries related to transnasal approach has been reported in some series to be approximately $2 \%$, including subarachnoid haemorrhage and intraventricular bleeding [16].

The cavernous sinus, a vascular landmark located in the parasellar space, the medial wall separates the pituitary fossa and is considered to be an important anatomic relationship related with sinus invasion by this tumours [17].

Cavernous sinus invasion has been reported to occur in $6-8 \%$ of patients with pituitary adenoma [18]. Since early descriptions of transsphenoidal anatomy, Harris and Rhoton have identified 4 different spaces of the cavernous sinus related to the internal carotid artery, in order to manage pathology compromising this vascular structures as well as for diminishing complications related to the approach; this spaces have been divided in anteroinferior, posterosuperior, medial and lateral [19].

Extended procedures through endoscopic approach and knowledge of anatomy has resulted in better outcomes and gross total resection of pituitary adenomas invading cavernous sinus, some authors have reported $81 \%$ of gross total tumour resection with no complications once cavernous sinus has been identified [20].

\begin{tabular}{|c|c|c|c|}
\hline Grade & $\mathbf{n}(\mathbf{\%})$ & Grade & $\mathbf{n}(\mathbf{\%})$ \\
\hline A & $6(6.12)$ & I & $1(1.02)$ \\
\hline B & $25(25.51)$ & II & $47(47.96)$ \\
\hline C & $35(35.71)$ & III & $26(26.54)$ \\
\hline D & $31(31.64)$ & IV & $24(24.48)$ \\
\hline E & $1(1.02)$ & & \\
\hline
\end{tabular}

Table 1: Distribution of patients according to Hardy - Vezina classification.

\begin{tabular}{|c|c|c|c|c|c|}
\hline Grade & $\mathbf{0}$ & $\mathbf{1}$ & $\mathbf{2}$ & $\mathbf{3}$ & $\mathbf{4}$ \\
\hline $\mathrm{n}$ & 17 & 16 & 15 & 39 & 11 \\
$(\%)$ & $(17.34 \%)$ & $(16.32 \%)$ & $(15.30 \%)$ & $(39.79 \%)$ & $(11.22 \%)$ \\
\hline
\end{tabular}

Table 2: Distribution of patients according to Knosp classification.

\begin{tabular}{|c|c|}
\hline Grade & $\mathbf{n}(\mathbf{\%})$ \\
\hline Partial $(<50 \%)$ & $11(11.22 \%)$ \\
\hline Subtotal $(50-75 \%)$ & $16(16.32 \%)$ \\
\hline Gross total $(75-90 \%)$ & $12(12.24 \%)$ \\
\hline Total $(>90 \%)$ & $59(60.20 \%)$ \\
\hline
\end{tabular}

Table 3: Grades of resection of pituitary adenomas through endoscopic endonasa approach. 
Citation: Gómez-Amador JL, Martínez-Anda JJ, Morales-Martínez AH, Llata-Segura CDL, Barges-Coll J, et al. (2015) Management of Complications in Endoscopic Endonasal Surgery for Pituitary Adenomas. J Neurol Disord 3: 209doi:10.4172/2329-6895.1000209

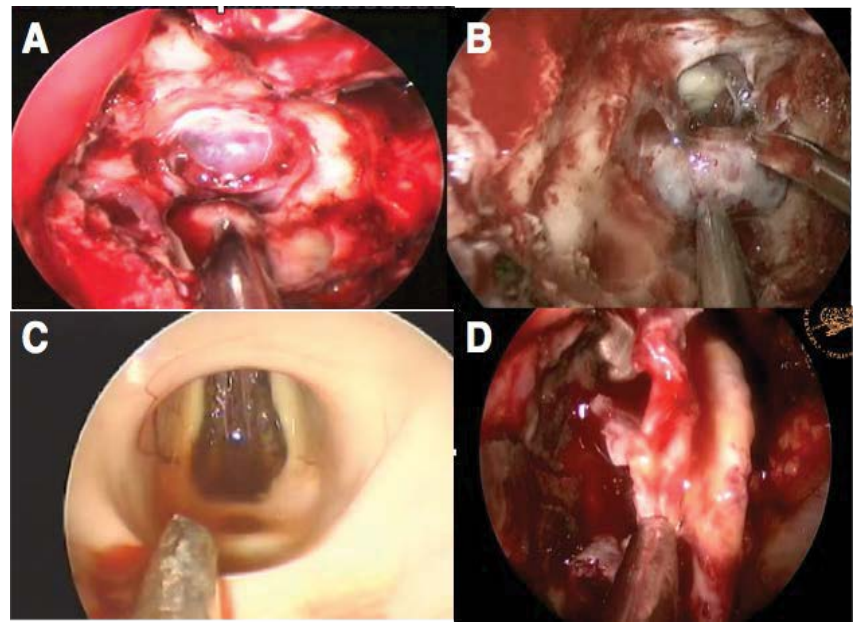

Figure 4: CSF leak risk in different situations A) Intact Arachnoid Membrane no fistula is expected; B) Suprasellar Cistern aperture, fistula is expected; C) Third Ventricle aperture, high risk of fistula; D) Endoscopic exploration of a patient with postoperative CSF leak; CSF:cerebrospinal fluid

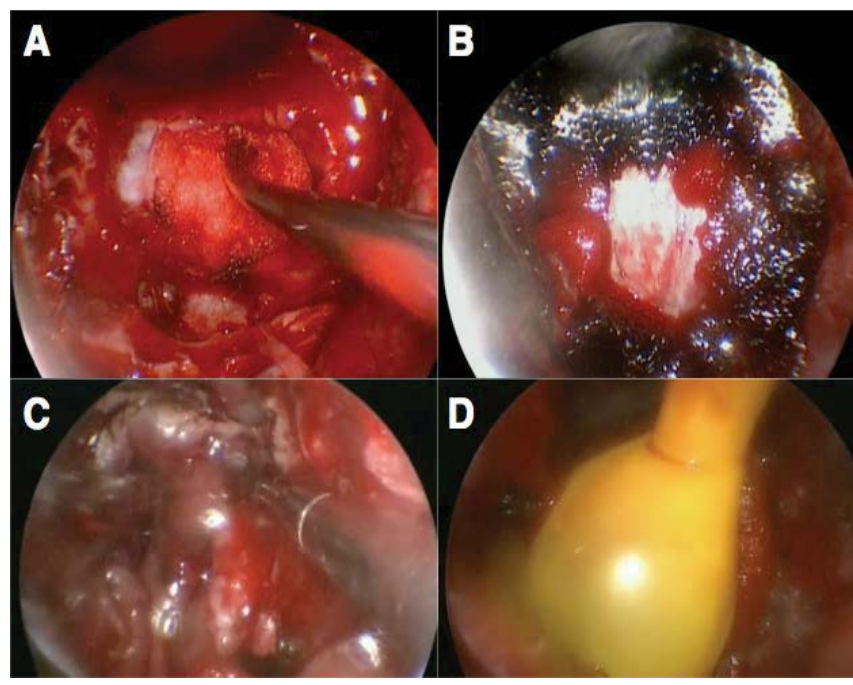

Figure 5: Sellar floor reconstruction after CSF leak. A) Duragen ${ }^{\circledR}$ patch placed intra- and extradurally. B) Nasal septum bone flap placed over the Duragen ${ }^{\circledR}$ patch. C) Nasoseptal pediculated flap placed over bone flap. D) Fixation of the reconstruction with Foley catheter, removed on postoperative day 5 . CSF=cerebrospinal fluid

The advantages of fully endoscopic procedures have been established by some authors comparing transsphenoidal and endoscopic procedures [21], In the series no vascular lesion, including patients with cavernous sinus compromise was evident. Also complications related to pituitary dysfunction were diminished via endoscopic approach to $2 \%$ as well as diabetes insipidus was seen in lower rates compared with transsphenoidal surgery.

The rate of vascular injuries in our series is $3.06 \%$ (3). When an intraoperative vascular injury happens we 1) identify the injury and control it with direct compression, 2) abdominal muscle is then harvested and a patch of a mixture of muscle and Surgicel ${ }^{\mathbb{}}$ is applied to the injury with compression over a period of 10 to 15 minutes, and 3) immediate angiographic control is performed with subsequent angiographies 3 and 6 month postoperative to assess for the risk of Carotid-Cavernous fistula development. Figure 6 shows types of vascular injuries found in patients operated endoscopically for pituitary adenomas.

The use of haemostatic agents have been described before with good results by several authors [22-24], in our centre, due to the demographics and economics of our population availability of different haemostatics is very limited, therefore routine use of this agents is not a common practice for our patients. When available we use BeriPlast ${ }^{\circ}$ (a biosurgical fibrin sealant kit) or Floseal ${ }^{\circledR}$ (a gelatine matrix haemostatic sealant)

\section{Cranial nerve injuries}

We describe two cases in which we had a cranial nerve injury. One was injury to the oculomotor nerve in which there was transitory deficit, and the other was an injury to the optic nerve with permanent deficit.

To prevent the occurrence of cranial nerve injuries, in patients with lesions invading the Cavernous Sinus with a Knosp classification $>2$ we performed intraoperative monitoring for $3 \mathrm{rd}$ and 6 th cranial nerves on the affected side.

\section{Conclusion}

The two major sources of morbidity in patients with skull base surgery are vascular injury and damage to cranial nerves. We consider cerebrovascular surgery techniques are of great importance as part of the armament that any skull base surgeon should have. Injury to the nerves in the skull base is associated with poor tolerance of these structures to manipulation. Therefore techniques must be implemented to avoid crossing the plane of the cranial nerves and sometimes multiple approaches considered so as to minimize at maximum the manipulation of these structures.

Ultimately the skull base surgery endonasal approach is a discipline that is constantly evolving with the advent of new techniques and the development of new technologies. This with the primary purpose to avoid damage, improve prognosis, reduce hospital stay and increase the quality of life of our patients. Analysis of the possible complications and planning strategies to prevent and treat them if necessary, are

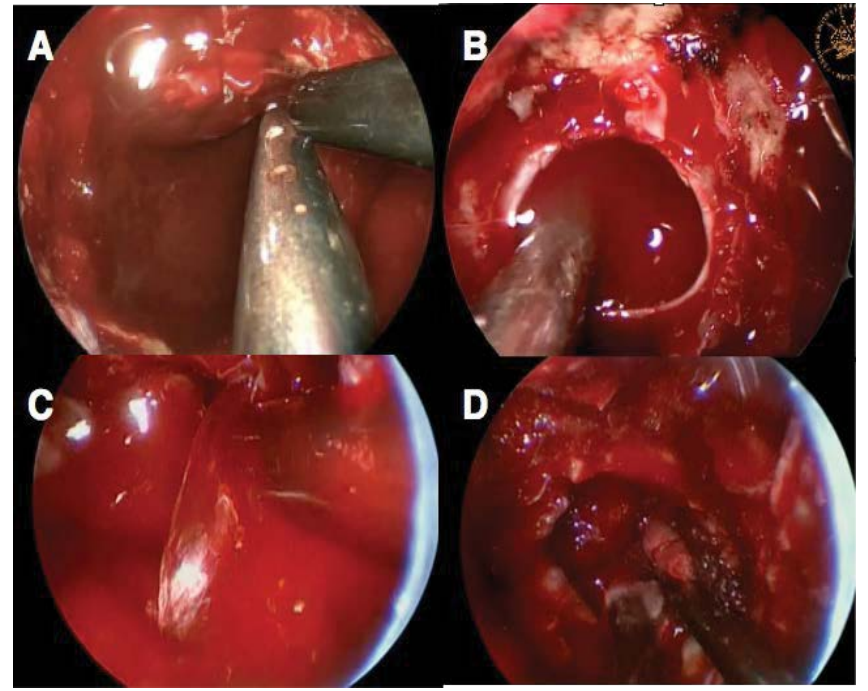

Figure 6: Types of Vascular Injuries A) Venous Haemorrhage from dura venous sinuses; B) Anterior Cerebral Artery branch bleeding; C) Internal Carotid Artery Injury; D) Vascular control with muscle patch over an Internal Carotid Artery Injury. 
Citation: Gómez-Amador JL, Martínez-Anda JJ, Morales-Martínez AH, Llata-Segura CDL, Barges-Coll J, et al. (2015) Management of Complications in Endoscopic Endonasal Surgery for Pituitary Adenomas. J Neurol Disord 3: 209doi:10.4172/2329-6895.1000209

main pillars to have an impact on long-term outcomes in patients with surgery for skull base pathology.

\section{References}

1. Cinalli G, Cappabianca P, de Falco R, Spennato P, Cianciulli E, et al. (2005) Current state and future development of intracranial neuroendoscopic surgery. Expert Rev Med Devices 2: 351-373.

2. Prevedello DM, Doglietto F, Jane JA Jr, Jagannathan J, Han J, et al. (2007) History of endoscopic skull base surgery: its evolution and current reality. J Neurosurg 107: 206-213.

3. Cappabianca P, Alfieri A, de Divitiis E (1998) Endoscopic endonasal transsphenoidal approach to the sella: towards functional endoscopic pituitary surgery (FEPS). Minim Invasive Neurosurg 2: 66-73

4. Alfieri A, Jho HD (2001) Endoscopic endonasal approaches to the cavernous sinus: surgical approaches. Neurosurgery 49: 354-360.

5. Hardy J, Vezina JL (1976) Transsphenoidal neurosurgery of intracranial neoplasm. Adv Neurol 15: 261-273.

6. Knosp E, Steiner E, Kitz K, Matula C (1993) Pituitary adenomas with invasion of the cavernous sinus space: a magnetic resonance imaging classification compared with surgical findings. Neurosurgery 4: 610-7- discussion 617-618.

7. Kassam $A B$, Prevedello DM, Carrau RL, Snyderman $C H$, Thomas $A$, et al (2011) Endoscopic endonasal skull base surgery: analysis of complications in the authors' initial 800 patients. J Neurosurg 114: 1544-1568.

8. Cavallo LM, Solari D, Esposito F, Cappabianca P (2012) Endoscopic endonasal approach for pituitary adenomas. Acta Neurochir (Wien) 154: 2251-2256.

9. Gondim JA, Almeida JP, Albuquerque LA, Gomes EF, Schops M (2014) Giant pituitary adenomas: surgical outcomes of 50 cases operated on by the endonasal endoscopic approach. World Neurosurg 82: e281-290.

10. Thomas JG, Gadgil N, Samson SL, Takashima M, Yoshor D (2014) Prospective trial of a short hospital stay protocol after endoscopic endonasal pituitary adenoma surgery. World Neurosurg 81: 576-583.

11. lannelli $A$, Lenzi $R$, Muscatello $L$ (2014) A useful maneuver to simplify sellar floor repair following endoscopic transnasal pituitary surgery. J Neurol Surg A Cent Eur Neurosurg 75: 158-160.

12. Cappabianca P, Cavallo LM, Esposito F, Valente V, De Divitiis E (2002) Sellar repair in endoscopic endonasal transsphenoidal surgery: results of 170 cases. Neurosurgery 51: 1365-1371.
13. Seda L, Camara RB, Cukiert A, Burattini JA, Mariani PP (2006) Sellar floo reconstruction after transsphenoidal surgery using fibrin glue without grafting or implants: technical note. Surgical Neurology 1: 46-49.

14. Zanation AM, Carrau RL, Snyderman CH, Germanwala AV, Gardner PA, et al. (2009) Nasoseptal flap reconstruction of high flow intraoperative cerebral spinal fluid leaks during endoscopic skull base surgery. Am J Rhinol Allergy 23: 518-521.

15. Güldner C, Pistorius SM, Diogo I, Bien S, Sesterhenn A, et al. (2012) Analysis of pneumatization and neurovascular structures of the sphenoid sinus using cone-beam tomography (CBT). Acta Radiol 53: 214-219.

16. Wang SS, Li JF, Chen HJ, Wang RM (2013) Clinical application of anatomy landmarks for microscopic endonasal transsphenoidal surgery for pituitary adenomas. J Craniofac Surg 24: 1785-1789.

17. Gonçalves MB, de Oliveira JG, Williams HA, Alvarenga RM, Landeiro JA (2012) Cavernous sinus medial wall: dural or fibrous layer? Systematic review of the literature. Neurosurg Rev 35: 147-153.

18. Ceylan S, Koc K, Anik I (2010) Endoscopic endonasal transsphenoidal approach for pituitary adenomas invading the cavernous sinus. J Neurosurg 112: 99-107.

19. Harris FS, Rhoton AL (1976) Anatomy of the cavernous sinus. A microsurgical study. J Neurosurg 45: 169-180.

20. Tabaee A, Anand VK, Fraser JF, Brown SM, Singh A, et al. (2009) Threedimensional endoscopic pituitary surgery. Neurosurgery 64: 288-293.

21. Kabil MS, Eby JB, Shahinian HK (2005) Fully endoscopic endonasal vs transseptal transsphenoidal pituitary surgery. Minim Invasive Neurosurg 48 348-354.

22. Padhye V, Valentine R, Paramasivan S, Jardeleza C, Bassiouni A, et al. (2014) Early and late complications of endoscopic hemostatic techniques following different carotid artery injury characteristics. Int Forum Allergy Rhinol 8: 651-657.

23. Speelman JP, Cahalane AK, Van Hasselt CA (2013) Evaluation of a porcine vascular model to assess the efficacy of various hemostatic techniques. J Invest Surg 26: 253-260.

24. Gardner PA, Tormenti MJ, Pant H, Fernandez-Miranda JC, Snyderman $\mathrm{CH}$, et al. (2013) Carotid artery injury during endoscopic endonasal skull base surgery: incidence and outcomes. Neurosurgery 2 Suppl Operative: ons 261-269. 